\title{
BMJ Open Protocol for a systematic review of instruments for the assessment of quality of life and well-being in children and adolescents with cerebral palsy
}

Christine Mpundu-Kaambwa, ${ }^{1}$ Gang Chen, ${ }^{2}$ Elisabeth Huynh, ${ }^{1}$ Remo Russo,,${ }^{3,4}$ Julie Ratcliffe ${ }^{1}$

To cite: Mpundu-Kaambwa C, Chen G, Huynh E, et al. Protocol for a systematic review of instruments for the assessment of quality of life and well-being in children and adolescents with cerebral palsy. BMJ Open 2017;7:e015924. doi:10.1136/ bmjopen-2017-015924

- Prepublication history and additional material for this paper are available online. To view please visit the journal (http:// dx.doi.org/10.1136/bmjopen2017-015924).

Received 10 January 2017 Revised 7 August 2017 Accepted 15 August 2017

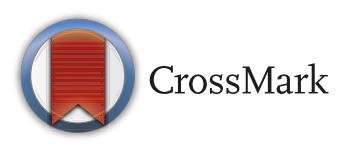

${ }^{1}$ Institute for Choice, University of South Australia Business School, University of South Australia, Adelaide, Australia

${ }^{2}$ Centre for Health Economics, Monash Business School, Monash University, Melbourne, Australia

${ }^{3}$ Faculty of Health Sciences, School of Medicine, Flinders University, Adelaide, Australia ${ }^{4}$ Department of Paediatric Rehabilitation, Women's and Children's Hospital, Adelaide, Australia

\section{Correspondence to}

Ms Christine Mpundu-Kaambwa; christine.mpundu-kaambwa@ unisa.edu.au

\section{ABSTRACT}

Introduction Cerebral palsy is the most common cause of physical disability in children and adolescents and is associated with impairments that may reduce the quality of life (QOL) of this population. Patient-reported outcome measures (PROMs) can facilitate the assessment of the effect of disease and treatment on QOL, from a patient viewpoint. The purpose of this systematic review is to identify PROMs that are used to measure QOL and subjective well-being (SWB) outcomes in young people with cerebral palsy and to evaluate the suitability of these PROMs for application in economic evaluations within this population.

Methods and analysis MEDLINE, Scopus, the Cochrane Library, Web of Science Core Collection, EconLit, PsycINFO, CINAHL, EMBASE and Informit will be systematically searched from inception to date of search. Published peer-reviewed, English-language articles reporting PROMs measuring QOL or SWB outcomes in children and adolescents with cerebral palsy will be included. One reviewer will conduct the initial search and screen titles and abstracts for potentially eligible studies. The search will be performed in November 2017. To reduce the likelihood of reviewer selection bias, two other reviewers will independently screen a randomly selected subsample (10\%) of the citations. Two reviewers will then retrieve full texts of potentially eligible studies and assess them against predefined inclusion criteria. The suitability of selected PROMs for use in economic evaluations of young people with cerebral palsy will be assessed using the International Society of Quality of Life Research recommended Minimum Standards and the Patient-Centered Outcomes and Comparative Effectiveness Research checklist. A narrative synthesis of extracted data will be presented including study descriptive data, PROMs measurement properties, settings in which they were applied and the valuation methods. Recommendations for practice on the selection of PROMs for use in economic evaluations of children and adolescents with cerebral palsy will be presented.

Ethics and dissemination Ethical approval is not required as the proposed systematic review will not use primary data. The results of this study will be widely

\section{Strengths and limitations of this study}

- One of the strengths of this study is that an extensive literature search of existing patient-reported outcome measures (PROMs) (both preference based and non-preference based) that are used to assess quality of life (QOL) in children and young people with cerebral palsy will be performed.

- Another strength of this systematic review is that a comprehensive examination of the suitability of preference-based PROMs for use within both trialbased and model-based economic evaluations of paediatric populations with cerebral palsy will be performed.

- A limitation of this systematic review is the exclusion of studies that are not published in English, which may mean that some articles examining QOL outcomes in young people with cerebral palsy in non-English-speaking countries maybe omitted.

disseminated through publication in a peer-reviewed journal and conference presentation(s).

Systematic review registration number International Prospective Register of Systematic Reviews number: CRD42016049746.

\section{INTRODUCTION}

Patient-reported outcome measures (PROMs) are increasingly being used in health services research to inform healthcare resource allocation decisions. ${ }^{12}$ PROMs assess a patient's subjective assessment of their well-being, health status or quality of life (QOL) at a single point in time and are collected via standardised, self-report questionnaires. ${ }^{3}{ }^{4}$ PROMs may be differentiated into condition-specific and generic measures. Condition-specific measures are designed to assess health outcomes in people with specific medical conditions (eg, the Cerebral Palsy 
Quality of Life questionnaire); while generic measures (eg, the Pediatric Quality of Life Inventory) are applicable across all disease areas. Condition-specific and generic measures can be subdivided into preference/utility-based PROMs and non-preference-based PROMs. Non-preference-based measures use a simple summative scoring system whereby individual items or dimensions are used to generate summary scores. ${ }^{5}$ Preference-based PROMs typically incorporate scoring algorithms which are premised on preferences of general population samples for health states generated through valuation methods such as the standard gamble) and time trade-off techniques, and are usually anchored between 0 (representing death) and 1 (representing optimal health). Preference-based PROMs enable the calculation of quality-adjusted life years (QALYs) for use in cost-utility analysis, a type of economic evaluation. ${ }^{5-7}$ QALYs are a routinely used standard measure of benefit in economic evaluation. ${ }^{8}$

Cerebral palsy is a complex chronic disorder of motor impairment that requires long-term medical and supportive care services. It is the leading cause of physical disability in childhood with prevalence rates ranging between 2.0 and 3.5 per 1000 live births worldwide. ${ }^{9}$ There are broad variations in the definition and classification of cerebral palsy. However, the International Executive Committee for the Definition of Cerebral Palsy recommend the following definition: 'Cerebral palsy describes a group of permanent disorders of the development of movement and posture, causing activity limitation, which are attributed to non-progressive disturbances that occurred in the developing fetal or infant brain. The motor disorders of cerebral palsy are often accompanied by disturbances of sensation, perception, cognition, communication and behaviour, by epilepsy and by secondary musculoskeletal problems' ${ }^{10}$ As such, cerebral palsy has ubiquitous impacts on all aspects of a child's life. Cerebral palsy has been shown to have a negative effect on the QOL of children with the condition. ${ }^{11} 12$ The cost of care for persons with cerebral palsy in Australia was estimated at AU $\$ 43431$ per person per year in 2007 with the total annual national economic cost of cerebral palsy estimated at AU $\$ 1.47$ billion (of which approximately $37 \%$ was borne by the individual and/or their family). ${ }^{13}$ In the USA in 2005, the total Medicaid expenditures averaged US $\$ 43338$ for a child with cerebral palsy ${ }^{14}$ and the average lifetime cost of cerebral palsy (based on 2003 US dollars) was estimated to be US\$921000 per person of which $81 \%$ are indirect costs and $19 \%$ are direct costs. ${ }^{15}$ In 2007, a Dutch study on children with cerebral palsy, found the annual cost to be $€ 40265$ per child. ${ }^{16}$ Clearly, it is vital to consider the impact of the cost of long-term care on young people and/or their families. With these rising costs and competing healthcare demands, there is a growing need for optimal funding decisions. Economic evaluation is an important technique to help decision makers determine the relative value for money of service innovations in healthcare and requires the robust measurement of appropriate health, health status, QOL or subjective well-being (SWB) outcomes. ${ }^{17}$ This highlights the importance of finding appropriate PROMs for economic evaluation of services targeted at children and young people with this condition.

The World Health Organisation defines QOL as 'an individual's perception of their position in life in the context of the culture and value system in which they live, and in relation to their goals, expectations, standards and concern'. ${ }^{18}$ QOL is a broad concept which refers to the influence of all facets of an individual's life on their general well-being including health-related QOL (HRQOL). HRQOL refers to an individual's self-perceived assessment of their health and its subsequent effect on their life and is defined as a subjective multidimensional construct of well-being and functioning based on physical, emotional, mental, social and behavioural features as perceived by patients. ${ }^{19}$ In literature these two concepts, QOL and HRQOL are used interchangeably, ${ }^{20}$ for the purposes of this systematic review both terms will be considered in the search strategy.

The main aim of this systematic review is to identify studies that have used PROMs to assess QOL and SWB in children with cerebral palsy and to evaluate the suitability of these PROMs for application in economic evaluations targeted at this population. Previous systematic reviews in cerebral palsy have focused on assessing performance of psychometric-based physical activity and/or participation measures ${ }^{21}{ }^{22}$ and QOL. ${ }^{23-25}$ These reviews did not distinguish between measures associated with different cerebral palsy health states depicting the levels of severity as classified using a number of metrics including gross motor function ${ }^{26}$ manual ability ${ }^{27}$ and communication. ${ }^{28}$ Further, only Janssens $e t a l^{25}$ included preference-based outcome measures in their review even though the population was that of children and young people living with neurological disabilities and not exclusive to those with cerebral palsy. This current review may be distinguished from previous ones in three main ways:

First, this review is focused exclusively on cerebral palsy. Second, the review will assess the appropriateness of the PROMs applied for informing QOL associated with different cerebral palsy health states for the purposes of model-based economic evaluation. Third, information on the contexts in which the PROMs have been used will also be extracted and collated so as to determine the suitability of particular PROMs for particular settings (with context defined according to the functional ability of populations as measured by the Gross Motor Function Classification System, ${ }^{26}$ in which the instruments have been used).

The specific objectives of the review are:

- To identify PROMs that are used to measure QOL and SWB outcomes in children and young people aged $0-18$ years with cerebral palsy.

- To establish the different contexts in which the PROMs have been applied.

- To critically examine the suitability of preference-based PROMs for use within economic evaluations targeted at this population. 


\section{Review questions}

The proposed review will seek to address the following specific research questions:

- What preference-based PROMs and non-preference-based PROMs are used to measure QOL and SWB outcomes in children and young people with cerebral palsy?

- How suitable are the identified PROMs for use within economic evaluations of paediatric populations with cerebral palsy and in what contexts?

\section{METHODS}

\section{Design}

This protocol has been registered with the International Prospective Register of Systematic Reviews (PROSPERO) (registration number CRD42016049746) and it has been developed using the Preferred Reporting Items for Systematic Review and Meta-Analysis Protocols (PRISMA-P) checklist. ${ }^{29}$ The review will be conducted in accordance with the PRISMA statement. ${ }^{30}$

The systematic review will follow a structured two-stage approach. First, all PROMs (both preference-based and non-preference-based) and articles/studies reporting details of development and/or application of PROMs used to measure QOL and/or SWB in young people with cerebral palsy will be identified. Second, each of the PROMs identified will be appraised using two checklists: The International Society of Quality of Life Research (ISOQOL) recommended Minimum Standards for Patient-Reported Outcome Measures, ${ }^{31}$ and the Patient-Centered Outcomes and Comparative Effectiveness Research (CREATE) checklist for reporting valuation studies. ${ }^{32}$ The ISOQOL was included for purposes of appraising non-preference-based and CREATE will be used to appraise the candidate utility-based (preference-based) PROMs. A University of South Australia Health Sciences Librarian with expertise in designing systematic reviews will be available to the team and will provide guidance on the search strategies for each database.

\section{Eligibility criteria}

Published, peer-reviewed, English-language articles reporting QOL and SWB outcomes of children and young people aged $0-18$ years with a diagnosis of cerebral palsy will be eligible for inclusion in the initial stage of the systematic review.

There is currently no consensus regarding the inclusion or exclusion of non-English-language articles to systematic reviews. Some authors seem to suggest that excluding non-English-language studies from systematic reviews may lead to language-bias and subsequently lead to inaccurate conclusions. However, other studies have reported results contrary to this which suggest that restricting searches to the English language does not alter the outcome of systematic reviews and meta-analyses. ${ }^{33} 34$ This is more so true in clinical fields which have a high prevalence of English-language articles and research.
Inclusion criteria

- The review will include all study designs in the preliminary search because it is expected that the number of preference-based PROMs in this field is relatively small.

- There will be no restrictions in terms of setting for the initial search because the literature in this field is likely to be relatively small and any restrictions at this stage over and above 'care-related' (rather than generic) would hinder any wider recommendations.

- To allow for a relatively broader definition of carers beyond primary carers, studies where PROMs were completed by either the child, parent (primary caregiver), clinician, teachers or school principal, among others will be included.

- Both preference and non-preference instruments will be included as well as generic and condition-specific instruments.

\section{Exclusion criteria}

- Studies focused only on adults. Studies that examine participants across both child/adolescent and adult age ranges will only be included if majority of the sample are children/adolescent and $0-18$ years and if results are for the two categories reported separately.

- Studies where QOL and SWB data are on parents and caregivers of children with cerebral palsy.

- Publications that are not peer-reviewed including unpublished dissertations, reports, conference presentations, discussion papers and any grey literature. This is so as to ensure that only articles that have gone through the rigorous review and editorial process are included.

\section{Search strategy}

An extensive search of the literature will be conducted in nine electronic bibliographic databases from database inception to the date of the search: MEDLINE (including in-process and other non-indexed citations via Ovid interface); Scopus (via Elsevier interface); The Cochrane Library (including the Cochrane CENTRAL, EED and HTA); Web of Science Core Collection; EconLit (via Ovid interface); EMBASE (via Ovid interface); PsycINFO (via Ovid interface); CINAHL (via EBSCO-host); Informit (via Informit interface). The primary electronic search strategy was designed for MEDLINE and adapted as appropriate for each of the databases. The full search strategy is presented in the online supplementary appendix. Keywords and Medical Subject Headings terms include: 'cerebral palsy', 'children', 'adolescents', 'quality of life', 'health related quality of life' and 'well-being'. To ensure that all significant literature is retrieved, both forward (inspecting articles in order to determine if key articles have been cited) and backward (examining reference lists) citation checking will be performed on all full texts examined so as to ensure that no eligible studies are missed out. Results from the search and retrieved references will be imported and managed in Thomson Reuters 
Endnote V.X7.1 (2014) reference management software. The search will be performed in November 2017.

\section{Selection process}

First, all titles and abstracts of articles resulting from the search will be screened against the eligibility criteria independently by the lead review author (CM), as has been done elsewhere. ${ }^{35-37}$ The primary aim of screening is to identify articles that meet the inclusion criteria. Full texts will be retrieved at this initial stage only if the abstract contains limited information about the study.All duplicate articles will be removed. To reduce the possibility of selection bias, a randomly selected subset of citations (20\%) will be independently assessed by two other members of the review team (GC and EH) ${ }^{37}$ Cohen's Kappa statistic will be estimated to measure inter-rater reliability (degree of agreement) between the reviewers. ${ }^{38-40}$ Cohen's Kappa statistic values less than or equal to 0 indicate no agreement, 0.01-0.20 (none to slight), 0.21-0.40 (fair), 0.410.60 (moderate), $0.61-0.80$ (substantial) and 0.81-1.00 (strong) agreement. If the inter-rater reliability is less than 0.80 , that is, strong level of agreement, ${ }^{39}$ an additional subset of articles (25\%) will be independently assessed. If the degree of agreement between the review authors is still less than 0.80 , then the rest of the articles will be independently screened. Differences will be resolved by discussion and consultation with the review team. Second, full texts of potential candidate studies will be obtained and assessed for inclusion in the review. To ensure that all relevant literature is retrieved, both forward (inspecting in order to determine if key articles have been cited) and backward (examining reference lists) citation chasing will be performed. Where necessary, study authors will be contacted for clarification and additional information to inform study selection. Each stage of the selection process will be outlined in a PRISMA-style flow chart and assessed against the 27-item PRISMA checklist.

\section{Data collection}

Summary data of each included PROM and article will be extracted into a data extraction form specifically designed for this review. Summary tables will be created in Microsoft Office Excel 2013 for (1) information about the candidate PROMs and (2) information pertaining to the identified studies. The information to be extracted from the included studies will be the following:

- Descriptive information about study: date of publication; country of origin; sample size; study type and setting; study population and characteristics (including age, gender and Gross Motor Functioning Classification System); study key results and conclusions.

- Descriptive information about the measure: name of PROM; domains/dimensions; number of items; description of the items; response method; method of administration; interpretation and summary scoring.

- Information about valuation of measure, that is, have preference weights been collected from a representative sample of children and adolescents with cerebral palsy? health states valued; preference elicitation method; population preference weights.

Two other reviewers will independently appraise the quality and suitability of the preference-based PROMs for measuring outcomes in paediatric populations with cerebral palsy. Any disagreements will be resolved by discussion and consultation with the review team. This evaluation will also follow the ISOQOL checklist (for internal consistency reliability, test-retest reliability, content validity, construct validity, criterion validity, responsiveness, interpretability of scores, respondent burden and investigator burden $)^{31}$ and the CREATE checklist (for reporting valuation studies) to appraise the candidate utility-based PROMs. ${ }^{32}$

\section{Data synthesis}

A summary of included studies and PROMs will be presented in line with recommendations from the Cochrane Collaboration. ${ }^{41}$ The main features of the included studies, instrument descriptions and contexts in which they are applied and information about valuation methods will be summarised into three tables. ${ }^{42}$ Using this information, the suitability of each of the PROMs identified for use in economic evaluation will be assessed and comparisons and disparities between instruments will be described.

\section{DISCUSSION}

To the best of our knowledge, this is the first systematic review that will comprehensively assess existing PROMs (both preference-based and non-preference-based) that are used to measure QOL in children and young people with cerebral palsy. Multiple bibliographic databases will be systematically searched from inception to date of search. This review will advance the field of health economics research in the following ways: first, the review will identify PROMs used to measure QOL and SWB in young people with cerebral palsy aged 0-18 years. Second, the review will establish the different contexts in which the PROMs have been applied. Third, the systematic review will provide evidence on the suitability of preference-based PROMs for use within both trial-based and model-based economic evaluations of paediatric populations with cerebral palsy.

A limitation of this systematic review is the exclusion of studies that are not published in English, which may mean that some articles examining QOL outcomes in young people with cerebral palsy in non-English-speaking countries maybe omitted. However based on results of previous reviews ${ }^{22} 24$ in this field as well as expertise and research experience of the research team, we do not anticipate a large number of non-English articles in this field and are therefore confident that no significant difference will be made by excluding them. 


\section{ETHICS AND DISSEMINATION}

The main aim of this review is to provide a systematic review of existing published literature and as such ethical approval to conduct this research is not required. This systematic review is registered with the PROSPERO (http:/ / www.crd.york.ac.uk/PROSPERO/display_ record.asp?ID=CRD42016049746), ${ }^{43}$ registration number CRD42016049746. The findings of this review will be disseminated as a peer-reviewed journal article and will be presented at both national and international conferences.

Contributors $\mathrm{CM}, \mathrm{JR}$ and $\mathrm{GC}$ formulated the idea for the study. CM wrote the first draft and the $\mathrm{co}$-authors (EH, GC, RR, JR) revised the protocol for important intellectual content. CM will act as a guarantor for the work.

Funding CM is supported by the Australian Government Research Training Program Scholarship.

Competing interests None declared.

Provenance and peer review Not commissioned; externally peer reviewed.

Open Access This is an Open Access article distributed in accordance with the Creative Commons Attribution Non Commercial (CC BY-NC 4.0) license, which permits others to distribute, remix, adapt, build upon this work non-commercially, and license their derivative works on different terms, provided the original work is properly cited and the use is non-commercial. See: http://creativecommons.org/ licenses/by-nc/4.0/

(C) Article author(s) (or their employer(s) unless otherwise stated in the text of the article) 2017. All rights reserved. No commercial use is permitted unless otherwise expressly granted.

\section{REFERENCES}

1. National Institute for Health and Care Excellence NICE. Guide to the methods of technology appraisal 2013. NICE 2013.

2. Deshpande PR, Rajan S, Sudeepthi BL, et al. Patient-reported outcomes: A new era in clinical research. Perspect Clin Res 2011;2:137-44.

3. Acquadro C, Berzon R, Dubois D, et al. Incorporating the patient's perspective into drug development and communication: an ad hoc task force report of the Patient-Reported Outcomes (PRO) Harmonization Group meeting at the Food and Drug Administration, February 16, 2001. Value Health 2003;6:522-31.

4. Willke RJ, Burke LB, Erickson P. Measuring treatment impact: a review of patient-reported outcomes and other efficacy endpoints in approved product labels. Control Clin Trials 2004;25:535-52.

5. Brazier J, et al. Measuring and valuing health benefits for economic evaluation. London: Oxford University Press, 2017.

6. Drummond MF SM, Torrance GW, O'Brien BJ, et al. Methods for the economic evaluation of health care programmes. Oxford: Oxford University Press, 2005.

7. Neumann PJ, Goldie SJ, Weinstein MC. Preference-based measures in economic evaluation in health care. Annu Rev Public Health 2000;21(1 1):587-611.

8. Brazier JE, Dixon S, Ratcliffe J. The role of patient preferences in cost-effectiveness analysis: a conflict of values? Pharmacoeconomics 2009;27:705-12.

9. Colver A, Fairhurst C, Pharoah PO. Cerebral palsy. Lancet 2014;383:1240-9.

10. Rosenbaum $P$, Paneth $\mathrm{N}$, Leviton $\mathrm{A}$, et al. A report: the definition and classification of cerebral palsy April 2006. Dev Med Child Neurol Supp/ 2007;109:8-14

11. Russo RN, Goodwin EJ, Miller MD, et al. Self-esteem, self-concept, and quality of life in children with hemiplegic cerebral palsy. J Pediatr 2008;153:473-7.

12. Livingston MH, Rosenbaum PL, Russell DJ, et al. Quality of life among adolescents with cerebral palsy: what does the literature tell us? Dev Med Child Neurol 2007;49:225-31.

13. Access Economics. The Economic Impact of Cerebral Palsy in Australia in 2007,6 Report for CP Australia. Canberra, Australia: Access Economics. 2008.

14. Kancherla V, Amendah DD, Grosse SD, et al. Medical expenditures attributable to cerebral palsy and intellectual disability among Medicaid-enrolled children. Res Dev Disabil 2012;33:832-40.
15. Centers for Disease Control and Prevention (CDC). Economic costs associated with mental retardation, cerebral palsy, hearing loss, and vision impairment--United States, 2003. MMWR Morb Mortal Wkly Rep 2004:53:57-9.

16. Hoving MA, Evers SM, Ament AJ, et al. Intractable spastic cerebral palsy in children: a Dutch cost of illness study. Dev Med Child Neurol 2007:49:397-8

17. Brazier J, Ratcliffe J, Salomon J, et al. Measuring and valuing health benefits for economic evaluation. London: Oxford University Press, 2007.

18. The world health organization quality of life assessment (WHOQOL). Position paper from the world health organization. Soc Sci Med 1995;41:1403-9.

19. Ravens-Sieberer U, Erhart M, Wille N, et al. Generic healthrelated quality-of-life assessment in children and adolescents: methodological considerations. Pharmacoeconomics 2006;24:1199-220.

20. Karimi M, Brazier J. Health, quality of life, and quality of life: what is the difference? Pharmacoeconomics 2016;34:645-9.

21. Harvey A, Robin J, Morris ME, et al. A systematic review of measures of activity limitation for children with cerebral palsy. Dev Med Child Neurol 2008;50:190-8.

22. Morris C, Kurinczuk JJ, Fitzpatrick R. Child or family assessed measures of activity performance and participation for children with cerebral palsy: a structured review. Child Care Health Dev 2005;31:397-407.

23. Davis E, Waters E, Mackinnon A, et al. Paediatric quality of life instruments: a review of the impact of the conceptual framework on outcomes. Dev Med Child Neurol 2006;48:311-8.

24. Carlon S, Shields N, Yong K, et al. A systematic review of the psychometric properties of Quality of Life measures for school aged children with cerebral palsy. BMC Pediatr 2010;10:81.

25. Janssens A, Rogers M, Gumm R, et al. Measurement properties of multidimensional patient-reported outcome measures in neurodisability: a systematic review of evaluation studies. Dev Med Child Neurol 2016;58:437-51

26. Palisano R, Rosenbaum $\mathrm{P}$, Walter $\mathrm{S}$, et al. Development and reliability of a system to classify gross motor function in children with cerebral palsy. Dev Med Child Neurol 1997;39:214-23.

27. Eliasson AC, Krumlinde-Sundholm L, Rösblad B, et al. The Manual Ability Classification System (MACS) for children with cerebral palsy: scale development and evidence of validity and reliability. Dev Med Child Neurol 2006;48:549-54.

28. Hidecker MJ, Paneth N, Rosenbaum PL, et al. Developing and validating the communication function classification system for individuals with cerebral palsy. Dev Med Child Neurol 2011;53:704-10.

29. Shamseer L, Moher D, Clarke M, et al. Preferred reporting items for systematic review and meta-analysis protocols (PRISMA-P) 2015 elaboration and explanation. BMJ 2015;349:97647.

30. Liberati A, Altman DG, Tetzlaff J, et al. The PRISMA statement for reporting systematic reviews and meta-analyses of studies that evaluate health care interventions: explanation and elaboration. PLoS Med 2009:6.

31. Reeve BB, Wyrwich KW, Wu AW, et al. ISOQOL recommends minimum standards for patient-reported outcome measures used in patient-centered outcomes and comparative effectiveness research. Qual Life Res 2013;22:1889-905.

32. Xie F, Pickard AS, Krabbe PF, et al. A checklist for reporting valuation studies of multi-attribute utility-based instruments (CREATE). Pharmacoeconomics 2015;33:867-77.

33. Morrison A, Polisena J, Husereau D, et al. The effect of Englishlanguage restriction on systematic review-based meta-analyses: a systematic review of empirical studies. Int J Technol Assess Health Care 2012;28:138-44.

34. Moher D, Pham B, Lawson ML, et al. The inclusion of reports of randomised trials published in languages other than English in systematic reviews. Health Technol Assess 2003;7:1-90.

35. Bhattarai N, McMeekin P, Price C, et al. Economic evaluations on centralisation of specialised healthcare services: a systematic review of methods. BMJ Open 2016;6:e011214.

36. Kigozi J, Jowett $\mathrm{S}$, Lewis $\mathrm{M}$, et al. Estimating productivity costs using the friction cost approach in practice: a systematic review. Eur $J$ Health Econ 2016;17:31-44.

37. McCaffrey N, Al-Janabi $\mathrm{H}$, Currow D, et al. Protocol for a systematic review of preference-based instruments for measuring care-related outcomes and their suitability for the palliative care setting. BMJ Open 2016:6:e012181.

38. Cohen J. A coefficient of agreement for nominal scales. Educ Psychol Meas 1960;20:37-46. 
39. Orwin. EG. L.H. Cooper CH, ed. Evaluating coding decisions, in The Handbook of Research Synthesis. New York: Russell Sage Foundation, 1994.

40. McHugh ML. Interrater reliability: the kappa statistic. Biochem Med 2012;22:276-82.

41. Ryan R. Consumers cochrane communication group: meta-analysis. 2016 http://cccrg.cochrane.org.
42. Green BN, Johnson CD, Adams A. Writing narrative literature reviews for peer-reviewed journals: secrets of the trade. J Chiropr Med 2006;5:101-17.

43. Mpundu-Kaambwa $\mathrm{CH}$, Chen E, Russo G, et al. A systematic review of quality of life outcome measures for children with cerebral palsy. PROSPERO International Prospective Register of Systematic Reviews 2016. 